\title{
The onset of spin rotation effects in electroproduction of heavy quarkonia
}

\author{
M. Krelina* \\ Universidad Tecnica Federico Santa Maria, Casilla 110, Valparaiso, Chile \\ Czech Technical University in Prague, FNSPE, Brehova 7, 11519 Prague, Czech Republic
}

E-mail: michal.krelina@usm.cl

\section{J. Nemchik}

Czech Technical University in Prague, FNSPE, Brehova 7, 11519 Prague, Czech Republic Institute of Experimental Physics SAS, Watsonova 47, 04001 Košice, Slovakia

\section{R. Pasechnik}

Department of Astronomy and Theoretical Physics, Lund University, SE-223 62 Lund, Sweden Nuclear Physics Institute ASCR, 25068 Rez, Czech Republic

Departamento de Física, CFM, Universidade Federal de Santa Catarina, C.P. 476, CEP 88.040-900, Florianópolis, SC, Brazil

\section{J. Cepila}

Czech Technical University in Prague, FNSPE, Brehova 7, 11519 Prague, Czech Republic

\begin{abstract}
We discuss the Melosh spin rotation effects in diffractive photo- and electroproduction of heavy quarkonia off a nucleon target in the dipole picture. The quarkonium light-front wave functions are obtained in the Schroedinger equation based formalism using the realistic interquark interaction potentials. A strong onset of spin rotation effects predicted in the case of $J / \psi(1 S)$ photoproduction enables us to reach a reasonable agreement with the available data. In the case of radially excited $2 S$ and $3 S$ states these effects are even stronger as a direct manifestation of the nodal structure of their wave functions. In particular, the spin effects enhance the $\Psi^{\prime}(2 S)$ cross section by a factor of $2-3$ and therefore cause a substantial increase of the $\Psi^{\prime}(2 S)$-to- $J / \Psi(1 S)$ ratio improving the description of experimental data.
\end{abstract}

European Physical Society Conference on High Energy Physics - EPS-HEP2019

10-17 July, 2019

Ghent, Belgium

${ }^{*}$ Speaker. 


\section{Introduction}

Electroproduction of heavy quarkonia is one of the most dynamic fundamental research areas for studies of various phenomena based on Quantum Chromodynamics (QCD). In comparison to production of other particles the heavy quarkonia represent an effective and cleaner probe for the medium created in heavy-ion collisions [1]. Particularly, in the present report we are interested in $S$-wave state production, $\gamma^{*} p \rightarrow\left(V=J / \psi, \psi^{\prime}, \Upsilon, \Upsilon^{\prime}, \ldots\right) p$ (for details, see Refs. [2, 3]).

One of the important ingredients in calculations of the production amplitude in the framework of the color dipole approach is the light-front (LF) wave function for heavy quarkonia. Its dependence on the interquark distance is strongly correlated with the form of the interquark $Q-\bar{Q}$ interaction potential. Although several realistic models for such potentials can be found in the literature (see e.g. Ref. [3]), many of the recent theoretical works still rely on an archaic parametrization for the quarkonia wave functions, the so-called Boosted Gaussian model [4] corresponding to a simple harmonic oscillator for the $Q-\bar{Q}$ potential in the rest frame. Moreover, the Coulomb repulsion at small $Q-\bar{Q}$ separations is frequently omitted, although this part of the potential was presented in the original publications [4].

The photon-like Lorentz structure for the transition $V \rightarrow Q \bar{Q}$ usually adopted in the literature leads to a conclusion that the transformation of spin-orbital part of the heavy quarkonium wave function from the rest to LF frame is effectively included. For this reason, the corresponding transformation is applied to the radial part only. However, such a photon-like structure effectively accounts for an admixture of a $D$-wave state that has not been observed experimentally (for more details, see Ref. [5]).

For this reason, in this report we consider a different, purely $S$-wave, structure for $V \rightarrow Q \bar{Q}$ amplitude [2,3]. The radial part of the quarkonium wave function is obtained in the rest frame by solving the Schroedinger equation with a realistic $Q-\bar{Q}$ interaction potential and by subsequent boosting of the corresponding solution to the LF frame. The same transformation is consistently applied also to the spin-orbital part; the procedure known as the Melosh spin rotation [6, 7]. We derive the corresponding formulae for the cross sections and study the onset of such spin transformation in electroproduction of heavy quarkonia in various kinematical regions.

\section{Quarkonia wave function}

The shape of the quarkonium wave function in the $Q \bar{Q}$ rest frame strongly depends on the choice of the $Q \bar{Q}$ potential $V_{Q \bar{Q}}$. There are several realistic potentials in the literature (see Ref. [3], for example). In the current report, we employ the Buchmüller-Tye potential [8], which has a linear string-like behavior at large transverse $Q \bar{Q}$ separations and a Coulomb shape at small separations.

Following the procedure developed in Ref. [7], the quarkonium wave function in the $Q \bar{Q}$ rest frame is obtained by solving the Schroedinger equation,

$$
\left(-\frac{\Lambda_{r^{\prime}}}{m_{Q}}+V_{Q \bar{Q}}\left(r^{\prime}\right)\right) \Psi_{n l m}\left(\vec{r}^{\prime}\right)=E_{n l} \Psi_{n l m}\left(\vec{r}^{\prime}\right)
$$

where $m_{Q}$ is the heavy quark mass, and the wave function $\Psi_{n l m}\left(\vec{r}^{\prime}\right)$ depends on 3-dimensional $Q \bar{Q}$ separation $\vec{r}^{\prime}$. The latter can be expressed in a factorized form,

$$
\Psi_{n l m}\left(\vec{r}^{\prime}\right)=\Psi_{n l}\left(r^{\prime}\right) \cdot Y_{l m}(\theta, \phi)
$$


where $\Psi_{n l}\left(r^{\prime}\right)$ and $Y_{l m}(\theta, \phi)$ represent the radial and spin-orbital part of the wave function, respectively. Typically, the Schroedinger equation is solved numerically for the radial part only, with the normalization condition $\int d^{3} r^{\prime}\left|\Psi\left(r^{\prime}\right)\right|^{2}=1$ applied.

According to the Terent'ev prescription [9], the wave function $\Psi\left(r^{\prime}\right)$ is first transformed into momentum representation,

$$
\Psi(p)=\frac{2}{\sqrt{2 \pi} p} \int_{0}^{\infty} d r^{\prime} r^{\prime} \Psi\left(r^{\prime}\right) \sin \left(p r^{\prime}\right), \quad \int d^{3} p|\Psi(p)|^{2}=1,
$$

and then boosted to the LF frame. This way, one finally obtains the following LF wave function $\Psi_{V}\left(p_{T}, z\right)[2,3]$,

$$
\Psi_{V}\left(p_{T}, z\right)=\left(\frac{p_{T}^{2}+m_{Q}^{2}}{16(z(1-z))^{3}}\right)^{1 / 4} \psi(p), \quad \int d^{2} p_{T} d z\left|\Psi_{V}\left(p_{T}, z\right)\right|^{2}=1
$$

where $z=p_{Q}^{+} / p_{\gamma}^{+}$is the boost-invariant fraction of the photon momentum carried by a heavy quark (or antiquark). Finally, the LF wave function for heavy quarkonia $\Psi(r, z)$ is obtained from $\Psi\left(p_{T}, z\right)$ by means of the corresponding Fourier transform. The Terent'ev prescription has recently been discussed and compared with exact calculations using the sophisticated Green function approach in Ref. [10].

Fig. 1 shows the LF wave functions for $J / \psi(1 S)$ (left panel) and $\psi^{\prime}(2 S)$ (right panel) states as functions of LF variables $r$ and $z$. One notices that the $\psi^{\prime}(2 S)$ wave function has non-monotonic $r$-behavior and contains a node.
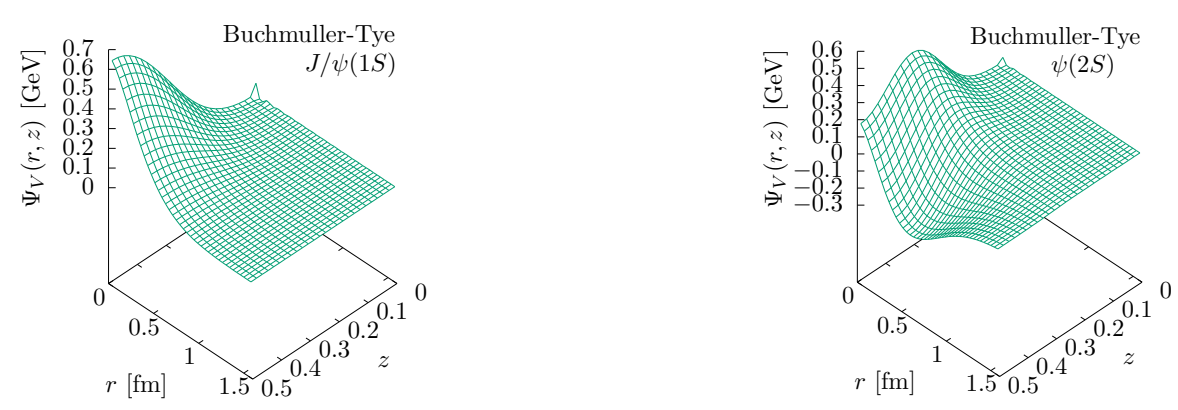

Figure 1: The LF wave functions for $J / \psi$ (left panel) and $\psi^{\prime}$ (right panel) calculated according to Eq. (2.4).

\section{Spin rotations in the dipole picture}

The exclusive quarkonium electroproduction amplitude can be written as [2, 3, 7]

$$
\operatorname{Im} A_{T, L}^{\gamma^{*} p \rightarrow V p}\left(x, Q^{2}\right)=\int d^{2} r \int_{0}^{1} d z \Psi_{V}^{\dagger}(r, z) \psi_{\gamma^{*}, L / T}(r, z) \sigma_{q \bar{q}}(x, r),
$$


where the cross section for the process $\gamma^{*} p \rightarrow V p$ reads,

$$
\sigma_{T, L}^{\gamma^{*} p \rightarrow V p}=\frac{1}{16 \pi B}\left|A_{T, L}^{\gamma^{*} p \rightarrow V p}\right|^{2}
$$

Here, $\Psi_{V}^{\dagger}(r, z)$ is the LF vector meson wave function; $B=4.73 \mathrm{GeV}^{-2}$ is the elastic slope parameter; $\psi_{\gamma^{*}, L / T}$ is the LF distribution for a $Q \bar{Q}$ fluctuation of a transversely (T) or longitudinally (L) polarized virtual photon, and $\vec{r}$ is the transverse size of the $Q \bar{Q}$ dipole. The universal dipole cross section $\sigma_{q \bar{q}}$ describes QCD interaction of the $Q \bar{Q}$ dipole (with transverse separation $r$ ) with a nucleon target.

The LF wave function $\Psi_{V}^{\dagger}(r, z)$ in Eq. (3.1) includes the spin rotation effects assuming a simple factorization of the spatial and spin-dependent parts of the vector meson wave function,

$$
\Psi_{V}^{\dagger}\left(\vec{p}_{T}, z\right)=U\left(\vec{p}_{T}, z\right) \psi_{V}\left(p_{T}, z\right), \quad U\left(\vec{p}_{T}, z\right)=\frac{1}{\sqrt{2}} \xi_{Q}^{\dagger}\left(\vec{\sigma} \cdot \vec{e}_{V}\right) \tilde{\xi}_{\bar{Q}}, \quad \tilde{\xi}_{\bar{Q}}=i \sigma_{y} \xi_{\bar{Q}}^{*}
$$

expressed in terms of the vector meson polarisation vector $\vec{e}_{V}$ and quark spinors $\xi$ in the meson rest frame. The latter are related to spinors $\chi$ in the LF frame as follows,

$$
\xi_{Q}=R\left(\vec{p}_{T}, z\right) \chi_{Q}, \quad \xi_{\bar{Q}}=R\left(-\vec{p}_{T}, 1-z\right) \chi_{\bar{Q}}
$$

Such a relation is known as the Melosh spin transformation [6, 7] with

$$
R\left(\vec{p}_{T}, z\right)=\frac{m_{Q}+M_{Q \bar{Q}}-i(\vec{\sigma} \times \vec{n}) \cdot \vec{p}_{T}}{\sqrt{\left(m_{Q}+z M_{Q \bar{Q}}\right)^{2}+p_{T}^{2}}},
$$

where $M_{Q \bar{Q}}=\sqrt{\left(p_{T}^{2}+m_{Q}^{2}\right) /(z(1-z))}$ is the invariant mass of the $Q \bar{Q}$ pair.

Following derivations in Refs. [2,3] one can obtain the following expressions for quarkonia photo- and electroproduction amplitudes in polarised photon-nucleon scattering,

$$
\begin{aligned}
\operatorname{Im} A_{L}^{\gamma^{*} p \rightarrow V p}\left(x, Q^{2}\right) & =\int d^{2} r \int_{0}^{1} d z \Sigma_{L}(z, r) \sigma_{q \bar{q}}(x, r) \\
\Sigma_{L} & =4 C Q z(1-z) K_{0}(\varepsilon) \int d p_{T} p_{T} J_{0}\left(p_{T} r\right) \Psi_{V}\left(z, p_{T}\right) \frac{m_{T} m_{L}+m_{Q}^{2}}{m_{Q}\left(m_{T}+m_{L}\right)} \\
\operatorname{Im} A_{T}^{\gamma^{*} p \rightarrow V p}\left(x, Q^{2}\right) & =\int d^{2} r \int_{0}^{1} d z\left[\Sigma_{T}^{(1)}(z, r) \sigma_{q \bar{q}}(x, r)+\Sigma_{T}^{(2)} \frac{d \sigma_{q \bar{q}}(x, r)}{d r}\right] \\
\Sigma_{T}^{(1)} & =2 C K_{0}(\varepsilon) \int d p_{T} p_{T} J_{0}\left(p_{T} r\right) \Psi_{V}\left(z, p_{T}\right) \frac{m_{T}^{2}+m_{T} m_{L}-2 p_{T}^{2} z(1-z)}{m_{T}+m_{L}} \\
\Sigma_{T}^{(2)} & =2 C K_{0}(\varepsilon) \int d p_{T} \frac{p_{T}^{2}}{2} J_{1}\left(p_{T} r\right) \Psi_{V}\left(z, p_{T}\right) \frac{m_{T}+m_{L}+m_{T}^{2}(1-2 z)^{2}}{m_{T}\left(m_{T}+m_{L}\right)}
\end{aligned}
$$

where $m_{T}^{2}=m_{Q}^{2}+p_{T}^{2}, m_{L}^{2}=4 m_{Q}^{2} z(1-z)$, and $C=Z_{q} \frac{\sqrt{N_{c} \alpha_{\mathrm{em}}}}{2 \sqrt{2} \pi}$. 


\section{Results}

The results of our calculations for $J / \psi(1 S)$ and $\psi^{\prime}(2 S)$ electroproduction cross sections are presented in Figs. 2 and 3. The calculations were performed using the KST [11] and GBW [12] parametrizations for the dipole cross section depicted by thin and thick lines, respectively. One observes that the Melosh spin transformation enhances the cross section and leads to a better agreement with the experimental data than in the case of no spin rotation.

The onset of spin effects is much stronger in production of radially excited $\psi^{\prime}(2 S)$ increasing significantly the electroproduction cross section by a factor of $2 \div 3$. This leads to a good agreement with the data for the $\psi^{\prime}$-to- $J / \psi$ production ratio as demonstrated in Fig. 3. A similar manifestation of the Melosh spin rotation is expected to emerge also for $\Upsilon(1 S)$ and $\Upsilon^{\prime}(2 S)$ [2, 3].

One can see from Fig. 2 that the onset of spin effects gradually decreases with the scale $Q^{2}+$ $M_{V}^{2}$. Such a behavior is expected due to a small $Q \bar{Q}$ dipole separation, as well as due to a dominance of $L$-polarised photon contribution to the electroproduction cross sections.

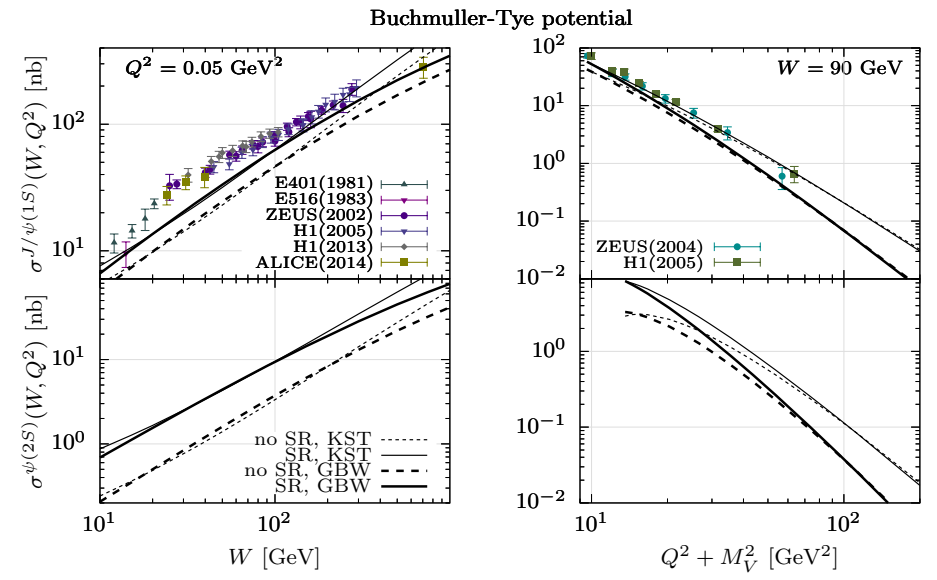

Figure 2: Predictions for the elastic cross sections $\sigma^{\gamma^{*} p \rightarrow J / \psi p}$ (upper panels) and $\sigma^{\gamma^{*} p \rightarrow \psi^{\prime} p}$ (lower panels) as functions of energy $W$ (left panels) and scale $Q^{2}+M_{V}^{2}$ (right panels).

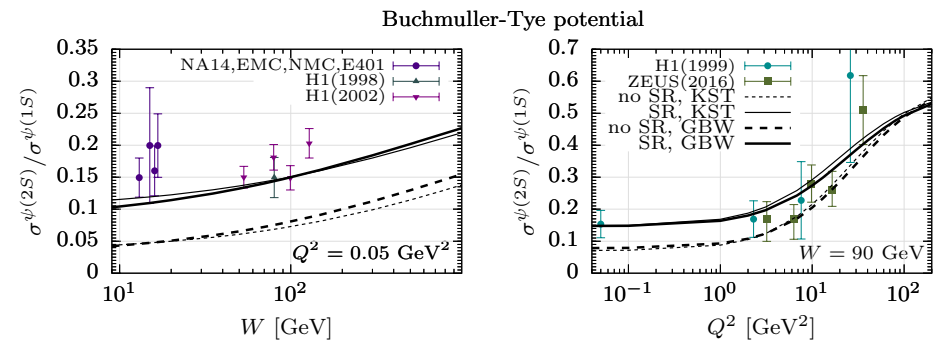

Figure 3: Predictions for the $\psi^{\prime}$-to- $J / \psi$ ratio of the electroproduction cross sections as functions of energy $W$ (left panel) and scale $Q^{2}+M_{V}^{2}$ (right panel).

Note that most of the results presented here, as well as in Refs. [2, 3], can also be obtained directly at our webpage: https://hep.fjfi.cvut.cz/vm.php. 


\section{Conclusions}

The main goal of this report was to demonstrate how to calculate the LF wave functions for $S$-wave heavy quarkonia using the realistic interquark interaction potentials in the $Q \bar{Q}$ rest frame and, consequently, to illustrate how to boost them to the LF frame using the popular Terent'ev prescription [9]. Considering the " $S$-wave only" structure for $V \rightarrow Q \bar{Q}$ transition in the $Q \bar{Q}$ rest frame we include the effects of the Melosh spin rotation boosting the spin-dependent part of quarkonium wave functions to the LF frame. The main new results can be formulated as follows:

i) Spin rotation effects contribute significantly to the observables and provide a reasonable agreement with the available data without any extra adjustable parameters;

ii) Spin rotation effects lead to a rise of the $\psi^{\prime}(2 S)$ photoproduction cross section by a factor of $2 \div 3$, thus causing a notable enhancement of the $\psi^{\prime}$-to- $J / \psi$ production ratio;

iii) Spin rotation effects gradually vanish at large $Q^{2}$.

\section{Acknowledgement}

The work of M.K. was supported in part by the Conicyt Fondecyt grant Postdoctorado N.3180085 (Chile) and by the grant LTC17038 of the INTER-EXCELLENCE program at the Ministry of Education, Youth and Sports of the Czech Republic. R.P. is supported in part by the Swedish Research Council grants, contract numbers 621-2013-4287 and 2016-05996, by the Ministry of Education, Youth and Sports of the Czech Republic, project LTC17018. J.N. work was partially supported by grants LTC17038 and LTT18002 of the Ministry of Education, Youth and Sports of the Czech Republic, by the project of the European Regional Development Fund CZ02.1.01/0.0/0.0/16_019/0000778, and by the Slovak Funding Agency, Grant 2/0007/18. The work has been performed in the framework of COST Action CA15213 "Theory of hot matter and relativistic heavy-ion collisions" (THOR).

\section{References}

[1] T. Matsui and H. Satz, Phys. Lett. B 178, 416 (1986).

[2] M. Krelina, J. Nemchik, R. Pasechnik and J. Cepila, Eur. Phys. J. C 79, no. 2, 154 (2019).

[3] J. Cepila, J. Nemchik, M. Krelina and R. Pasechnik, Eur. Phys. J. C 79, no. 6, 495 (2019).

[4] J. Nemchik, N.N. Nikolaev and B.G. Zakharov, Phys. Lett. B 341 (1994) 228; J. Nemchik, N.N. Nikolaev, E. Predazzi and B.G. Zakharov, Z. Phys. C 75 (1997) 71; H. Kowalski, L. Motyka, G. Watt, Phys. Rev. D 74 (2006) 074016.

[5] M. Krelina, J. Nemchik and R. Pasechnik, arXiv:1909.12770 [hep-ph].

[6] H.J. Melosh, Phys. Rev. D 9, 1095 (1974).

[7] J. Hufner, Y.P. Ivanov, B.Z. Kopeliovich, A.V. Tarasov, Phys. Rev. D 62, 094022 (2000)

[8] W. Buchmuller, S.H.H. Tye, Phys. Rev. D 24, 132 (1981).

[9] M.V. Terentev, Sov. J. Nucl. Phys. 24, 106 (1976). [Yad. Fiz. 24,207 (1976)].

[10] B.Z. Kopeliovich, E. Levin, I. Schmidt, M. Siddikov, Phys. Rev. D 92, 034023 (2015).

[11] B.Z. Kopeliovich, A. Schäfer, A.V. Tarasov, Phys. Rev. D 62, 054022 (2000)

[12] K. Golec-Biernat, M. Wüsthoff, Phys. Rev. D 53, 014017 (1999) 